\title{
COMPETENCIAS REQUERIDAS PARA EL EJERCICIO DE LAS PROFESIONES DE LA INFORMACIÓN: VALORACIÓN DE LAS LISTAS RELACIONALES DE MERCOSUR Y DE LA UNIÓN EUROPEA
}

\author{
José Antonio Moreiro \\ Carlos Miguel Tejada Artigas
}

\section{Resumo}

A Sociedade da Informação exige novas atitudes e desenvolvimento de competências necessárias para o bom desenvolvimento profissional, tendo como referência as profissões que trabalham com informação, assim como em relação às necessidades empresariais referentes ao tratamento da informação que necessitam e utilizam. As competências requeridas hoje, especificamente na área da Ciência da Informação, são necessidades a serem contempladas pela formação universitária, principalmente nos cursos de graduação. São apresentadas no texto as competências profissionais em Biblioteconomia e Documentação, de acordo com recomendações do Conselho da Europa; as carências observadas na área; as competências definidas para o Mercosul e a União Européia; assim como um projeto para o desenvolvimento dessas competências, conhecido pela sigla DECIDOC.

\section{Palavras-Chave}

Competências; Profissional da Informação; Mercosul; União Européia; DECIDOC. 


\section{INTRODUCCIÓN}

Metidos en una sociedad de la información, donde la flexibilidad y la adaptación juegan un papel fundamental, la universidad no puede quedarse fuera de los procesos de cambio. ${ }^{1}$ Tendrá que superar su tradicional continuismo y acometer sin lugar a dudas las medidas de mejora y transformación que se requieran. Pero alcanzar este punto no es una tarea simple, es necesario realizar antes un período de análisis y retlexión en el que participen los diferentes colectivos afectados em la formación universitaria: alumnos, profesores, y responsables académicos; profesionales y empleadores; y hasta seria conveniente que participasen los representantes sociales y quienes definen las políticas educativas.

Esta situación es especialmente notaria en el área de la Ciencia de la Información, pues si algo cambia con rapidez en esta sociedad es la información y cuanto se refiere a su acceso y control, sin duda por efecto de la intervención tecnológica de los ordenadores y las telecomunicaciones, que han modificado radicalmente las formas de acceso a la información. Las llamadas tecnologías de la información se han convertido en el factor más importante no sólo de

\footnotetext{
${ }^{1}$ A partir del momento social y económico Kutscher (1990) justificó el declive de algunas profesiones, y la necesidad de formación de nuevos profesionales en trabajos de nueva y creciente demanda.
}

la transformación de las tareas profesionales de la información, sino incluso de la concepción y funcionamiento de los sistemas de información (TENNANT, 1999). Las profesiones documentales han acusado con gran sensibilidad, y continúan haciéndolo, el tremendo impacto de las tecnologías inforrnáticas y de las telecomunicaciones. La aplicación de estas en pocos sectores laborales ha modificado tanto los sistemas y los métodos de trabajo. Esta nueva situación y las tendencias previsibles vienen delimitadas por las competencias que necesitan los nuevos profesionales. Máxime si consideramos que con las competencias se asocian factores críticos como la acreditación, los modelos nacionales, la certificación, la educación y formación, la promoción profesional, la formación permanente, y las cualidades y antecedentes personales. (COOPER; LUNIN, 1989).

En la actualidad los estudios sobre la profesión muestran una gran actividad. En especial en torno al análisis de las competencias exigidas para los puestos de trabajo y de las estrategias destinadas a lograr especialistas bien cualificados. El incentivo para conseguirlo se ha debido en gran parte al reto que sobre la reforma de la formación se iniciá en Estados Unidos com el American 2000 (1991). En las competencias exigibles a quien deba desarrollar su actividad profesional en el campo de la Ciencia de la Información, han tenido un im- 
pacto considerable las aplicaciones de las tecnologías de la información a las tareas profesionales, especialmente en la creación y procesamiento de los contenidos. El rápido desarrollo y utilización tecnológicos ha transformado a los profesionales de la información en: (DAVENPORT; PRUSAK, 1998)

- Analistas y filtradores de la información, con lo que agregan valor a los contenidos;

- Bibliotecarios de investigación, al seleccionar y reunir información;

- Buscadores de la información pertinente en el momento preciso;

- Catalogadores y archiveros que se sirven de métodos electrónicos;

- Expertos en tecnología, para cuyo uso forman y estimulan desde su puesto de trabajo;

- Informadores que ayudan a los usuarios a comprender lo que ellos han aprendido previamente;

- Orientadores en el uso de los recursos electrónicos correctos.

Pero también otros factores se han visto implicados en los cambios que la sociedad de la información ha supuesto para los profesionales de la información, como son: (WARD, 1999)

- El acceso general a la información;
- La aceleración de los cambios por la innovación de productos y procesos;

- El aumento de la competitividad;

- La diferencia competitiva entre información y conocimiento;

- La explosión informativa continua.

Sin duda alguna, el futuro de los profesionales de la información dependerá del valor que sean capaces de añadir a la institución o organización en la que trabajen. En los servicios de información se cuidarán especialmente cuatro aspectos relacionados con: (BARDEN, 1997)

- Las habilidades tecnológicas y la gestión de redes de comunicación;

- La atención preferente ai cliente;

- El almacenamiento, gestión y recuperación de los distintos soportes de información;

- El desarrollo empresarial.

Precisamente en el ámbito de la Ciencia de la Información viene siendo común que se determine de manera exacta cuál es la preparación con que los graduados deben salir de las aulas, es decir, qué nível de conocimientos y de habilidades deben poseer para enfrentarse a los requerimientos de unos puestos de trabajo cada vez más complejos por la variedad de funciones a cubrir y por las actividades que tienen que desarrollar (MOREIRO, 1998). Los profesionales necesitan habilidades 
para concentrar, analizar, e interactuar con los conocimientos. Con el fin de poder comunicarse sobre la estructura y acceso a la información en un entorno laboral cambiante. Estando inmersos en este medio basado en la información, en especial como profesionales, debemos aceptar la transformación del trabajo y de las organizaciones como una dialéctica perpetua. El esfuerzo de adaptación debe hacerse de forma concurrente y cooperativa por las empresas, las instituciones educativas, el gobierno y las asociaciones, para determinar si los trabajadores actuales poseen estas habilidades, y para fomentar estrategias que las desarrollen.

En la descripción del panorama de competencias que deben adquirir quienes deseen desempañar con acierto sus actividades profesionales, que es el objeto de estas páginas, nos hemos propuesto alcanzar los siguientes objetivos:

- Constatar las competencias y actitudes de un profesional de la infor-mación en MERCOSUR y en la Unión Europea, que tiene como resultado la posterior orientación de la formación universitaria hacia esas habilidades necesarias;

- Identificar los principales requerimientos que esperan a los titulados a la llegada al mundo de trabajo por parte de las empresas e instituciones que les emplean;
- Detectar indirectamente las características que debe poseer la formación de los profesionales de la información para realizar adecuadamente sus funciones laborales de acuerdo con las competencias que se suponen deben caracterizarles. ${ }^{2}$

Para hacerlo nos hemos propuesto una trayectoria consistente, primero, en considerar los principales trabajos que se han referido a las competencias, asi como los resultados de una observación aplicada al nivel de conocimientos y habilidades que el mercado de trabajo há requerido a los licenciados en Documentación por la Universidad Carlos III de Madrid. Para después valorar las competencias y aptitudes que las asociaciones profesionales e instituciones de MERCOSUR y de la Unión Europea consideran más relevantes para ejercer com éxito los requisitos profesiona-

\footnotetext{
${ }^{2}$ Son muy numerosos los estudios que se han dedicado sobre todo en Estados Unidos al análisis de las relaciones entre universidad, empleo y sociedad. Hemos de ver una monografia de Cronin como la principal referencia de los estudios que relacionan la profesión con la formación. Se hizo para identificar las tendencias más significativas en los planes de estudio existentes. A la par que consideraba la futura función de las Escuelas de Biblioteconomía para satisfacer las necesidades educativas en el momento en que la sociedad de la información emergía. Propuso directrices para diseñar los planes de estúdio en un momento de cambio y de autocritica profesional. Para lo cual planteó los interrogantes fundamentales sobre la educación más adecuada que debían recibir los futuros profesionales de la información, en qué instituciones, cómo se rediseñarían los planes de estudio, y cómo podían ser evaluados. La solución venía de la relación com las condiciones externas, como son las políticas de educación, las exigencias del mercado profesional, la innovación tecnológica, los valores sociales, y las preferencias de los estudiantes (CRONIN, 1983)
} 
les de quienes se dedican a la documentación e información.

\section{2 ¿QUÉ DEBEMOS ENTENDER POR COMPETENCIA?}

Nos referimos como competencias a aquellas capacidades que son necesarias para ejercer una actividad profesional, junto a los comportamientos en un puesto de trabajo. EI IV Encuentro de Directores de Escuelas de Bibliotecología y Ciencia de la Información del MERCOSUR, celebrado en Montevideo en mayo de 2000, recogió el reto de definir lo que debe entenderse por competencias profesionales. Las comprendió como

el conjunto de habilidades, destrezas y conocimientos que requiere contar un profesional en cualquier disciplina, para cumplir com su actividad especializada ofreciendo un mínimo de garantía sobre los resultados de su trabajo, tanto a los clientes y empleadores como, en última instancia, a la sociedad de la que forma parte. (VALENTIM, 2000, p. 17).

Así pues, las competencias son el conjunto de conocimientos, destrezas y aptitudes cuya finalidad es la realización de actividades definidas y vinculadas a una determinada profesión.

Ser competentes respecto al desarrollo de una actividad profesional significa poseer al mismo tiempo conocimientos y aptitudes, Por lo que atañe de modo específico a nuestra especialidad, esta dualidad que- dó explicitada cuando la Special Libraries Association (1996) redactó las competencias que debían poseer los bibliotecarios especializados del siglo XXI:

- Los conocimientos profesionales se relacionan con los recursos que deben caracterizar a los bibliotecarios especializados y con la habilidad de utilizar esos conocimientos a la hora de proporcionar los diferentes servícios;

- mientras que las aptitudes personales se refieren a habilidades, actitudes y valores que permiten a los bibliotecarios trabajar de forma eficiente en el nuevo panorama de trabajo, son el proceso por el que cada persona pone en acción sus conocimientos de una forma distinta. En definición incluída en las Eurocompetencias en Información y Documentación las aptitudes son consideradas como "las disposiciones, naturales o adquiridas, que provocan una manera de comportarse" (SEDIC, 2000, p. 14). Cada profesión tiene una serie de aptitudes, a veces compartidas, que inducen a los diferentes comportamientos.

En nuestra preocupación como profesores universitarios en el campo de la información está permanentemente presente la relación entre preparación académica y actividad profesional, que por sí misma ocupa el espacio principal de las investiga- 
ciones sobre competencias y mercado laboral en Estados Unidos. ${ }^{3}$

\section{LAS LISTAS DE RELACIONES SOBRE} COMPETENCIAS PROFESIONALES EM BIBLIOTECONOMÍA Y DOCUMENTACIÓN

Las comunicaciones, las relaciones humanas y la capacidad de adaptarse a las nuevas tecnologías han solido aparecer, de forma reiterada, como las competencias más necesitadas por los profesionales de la información. En el afán de identificar estas se ha llegado, incluso, a comparar las percepciones que a este respecto tenían los profesores y los profesionales de la Biblioteconomía y Documentación. Encotrandose claras diferencias en el concepto de troncalidad poseído por unos y por otros (SCHLESSINGER; SCHLESSINGER; KARP, 1991). En los últimos años se han diseñado una serie de proyectos con objeto de identificar, definir y sistematizar qué conocimientos, habilidades, aptitudes, y hasta actitudes, deben caracterizar al profesional de la información y documentación. Los interesados han sido generalmente asocia-

\footnotetext{
${ }^{3}$ En este sentido, Carol Leannont y Stephen Van Houten (1990) compilaron y analizaron los datos extraídos del 39 informe anual del ALA de 1989, y lo compararon con los datos de 1988. Pudieron apreciar cómo habían crecido los salaríos, qué puestos iban ocupando los titulados, y qué situaciones especiales se observaban. En su informe se destacó un aumento en la demanda sobre la oferta en catalogación, servicios de atención a niños y jóvenes, bibliotecas médicas y jurídicas, automatización, y publicaciones oficiales.
}

ciones profesionales, o otras instituciones que forman parte activa en esta profesión, o tienen provecho e influyen en ella. Hacemos una relación muy abreviada de los listados más representativos, aparte de aquellos cuyo análisis detallaremos luego. ${ }^{4}$

\subsection{Competencias de los Profesionales} de la Información Recomendadas por el Consejo de Europa

Las Recomendaciones del Consejo de Europa sobre perfiles y competencias de los profesionales de la información y del conocimiento que trabajan en las industrias e instituciones culturales pretenden

mantener una política cultural orientada a las nuevas tecnologías para animar el desarrollo de las industrias culturales e instituciones asegurando que sus profesionales de información y proveedores del contenido estén listas para participar totalmente en la nueva cadena de información (COUNCIL OF EUROPE, 1999).

Las competencias que aconseja se clasifican así:

1. Habilidades directivas:

- Habilidades analíticas, para combinar y organizar la información compleja, a fin de contribuir a la generación de ideas innovadoras;

- Habilidades directivas que capaciten para emprender proyectos con êxito;

\footnotetext{
${ }^{4}$ Hace una excelente tarea de síntesis sobre estos listados Tejada Artigas (2002).
} 
- Capacidad para generar estrategias comerciales basadas en el razonamiento económico;

- Habilidades comerciales, que consideren las exigencias del cliente, un mayor conocimiento del mercado y los objetivos estratégicos a largo plazo;

- Habilidades gestoras de los sistemas por objetivos hacia el logro de metas específicas.

2. Habilidades organizativas:

- Capacidad de formar, coordinar y dirigir equipos;

- Flexibilidad en la organización de los equipos de trabajo;

- Capacidad de crear y organizar un servicio para al público, entendiendo sus demandas.

3. Capacidades creativas:

- Puesta en marcha de planificaciones innovadoras que puedan asumir proyectos aplicables a nuevos productos 0 servicios;

- Capacidad para desarrollar ideas de una manera independíente, autónoma y comprometida.

4. Informática:

- Habilidades técnicas y comprensión profunda de los programas de ordenador para dominar un proceso técnico cambiante;

- Dominio de las técnicas multimedia;

- Conocimiento profundo de normas, formatos y métodos normalizados de descripción, presentación, y transmisión.

- Habilidad para navegar por las redes de comunicación mediante motores de búsqueda y otras herramientas.

5. Actitudes personales:

- Habilidades cognoscitivas entre las que se incluye la flexibilidad hacia las nuevas tareas profesionales. Esto conlleva la capacidad de aprendizaje a lo largo de toda la vida.

6. Habilidades legales:

- Habilidades legales aplicables a cualquier aspecto de la creación y difusión de los nuevos medios de comunicación electrónicos.

7. Habilidades de comunicación:

- Capacidad de comunicación;

- Capacidad de transferencia del conocimiento.

8. Habilidades lingüísticas:

- Habilidad de expresarse en uno o más idiomas de comunicacíón internacional. 


\subsection{Habilidades para la Gestión del Co-} nocimiento

El Institute of Information Scientists de Inglaterra elaboró una relación con las habilidades necesarias para ejercer esta profesión. Determinar las competencias serviría además para que se pudiesen acreditar sus cursos. Estructuraron estas habilidades así: (2003)

- Área básica de Ciencia de la Información: características de la información, sus proveedores y usuarios, fuentes de información, recuperación y almacenamiento de la información, análisis de la información, y teoria de la ciencia de la información;

- Gestión de la información: planificación, comunicación, sistemas de gestión y control de la información, gestión de recursos humanos, gestión financiera, promoción, economía y marketing, y factores políticos, éticos, sociales y legales;

- Tecnología de la información: sistemas informáticos: hardware y software, telecomunicaciones, aplicaciones de tecnología de la información, y cuestiones del entorno;

- Habilidades auxiliares: técnicas de investigación, lingüística e idiomas.

\subsection{EI Proyecto Futureprof}

EL proyecto Futureprof (EUROCHAMBERS, 1999) se propuso fomentar en Europa las ocupaciones que ofreciesen un futuro prometedor, por basarse en el desarrollo de la sociedad de información y en las nuevas tecnologías. Para los intermediadores de la información dibujó un perfil delimitado por estas funciones:

- Usa tecnologías de la información.

- Busca información.

- Interpreta información y compila documentos.

- Valida la información según las necesidades del cliente.

- Presenta la información.

- Usa y produce estadísticas.

- Usa bases de datos e Internet.

- Analiza las necesidades del cliente.

- Planifica proyectos.

- Ejecuta proyectos.

- Colabora en esos proyectos en equipo.

- Determina prioridades de trabajo y horarios.

- Documenta su propio trabajo.

- Resuelve problemas.

- Disena o produce creativamente.

- Piensa en conexiones complejas. 
- Tiene una actitud de aprendizaje permanente hacia las innovaciones.

- Realiza presentaciones.

- Coopera y comunica en equipos.

- Trabaja y se comunica con los clientes.

- Se organiza su propio trabajo eficazmente.

- Participa en la gestión de calidad.

- Se comunica en lenguas extranjeras.

\subsection{EI Pensamiento de Diversos Autores sobre las Competencias}

Otra relación, en este caso con los conocimientos y competencias propios de los bibliotecarios, nos la dio Maurice Line. Como trabajadores del sector terciario los bibliotecarios tienen que caracterizarse por poseer unas competencias comunes con los profesionales de ese sector respecto a conocimientos generales, Economía, Sociología, Psicología, Ética, y Gestión (LINE, 1998).

Mientras que comprendió como competencias específicas de los bibliotecarios:

A) Tecnología:

- Comunicaciones.

- Informática.

B) Gestión de la información:
- Conocimiento de los recursos documentales.

- Elaboración y transferencia de la información.

- Organización de los conocimientos.

- Catalogación.

- Lenguajes documentales.

C) Edición:

- Composición de las obras.

- Historia.

- Sector editorial.

- Mercado del libro.

De otra parte, mientras que una aportación suiza prefirió fijarse en las aptitudes (DESCHAMPS, 1999), Dominique Arot (2000) analizó algunos estudios de identificación y valoración de competencias realizados en Francia. Entre ellos uno sobre las competencias requeridas en las ofertas de empleo por las bibliotecas municipales $y$ departamentales francesas. $Y$ otro sobre el informe dirigido por Anne Kupiec titulado Prèmier recensement des métiers des bibliothèques, en el que se identificaron 27 competencias ligadas al trabajo de los bibliotecarios públicos (AROT, 2000).

\subsection{Uma Aproximación Española al Marco Competencial Profesional}

Finalmente, comentamos una parte de los resultados de un trabajo aplicado 
desde el que se pueden determinar las competencias exigibles a quien desee desarrollar su actividad en el campo de la información. Para hacerlo es conveniente que dibujemos las líneas principales en que se desarrolla la actividad profesional en ese campo. Utilizamos aquí una medición hecha a los alumnos del último año de la Licenciatura en Documentación en la Universidad Carlos III de Madrid durante los anos 1997-2000. ${ }^{5}$ De los resultados obtenidos, haremos constar, primero, aquellos sectores de actividad en los que obtuvieron empleo, para luego ver qué exigencias pedían las empresas e instituciones a los alumnos que iban a contratar, así como comentar el nível de adecuación entre preparación académica y exigencias de trabajo por parte tanto de las empresas como de los propios universitarios.

Respecto al análisis sectorial de las empresas e instituciones que han contratado a nuestros estudiantes durante ese periodo, hay que resaltar que es interesante porque marca las tendencias bacia las que se dirigen los sectores de actividad más vinculados com las actividades de la información documental. De manera que pueden considerarse como un reflejo de la penetración profesional de nuestra profesión. El seguiente cuadro hace referencia al sector de actividad de las empresas que han contratado a los alumnos, mencionando también el número de puestos ofertados en cada sector:

\begin{tabular}{|l|c|}
\hline \multicolumn{1}{|c|}{ Sector de Actividad } & $\begin{array}{c}\mathbf{N}^{\mathbf{0}} \text { de pues- } \\
\text { tos ofertados }\end{array}$ \\
\hline Consultoria & 35 \\
\hline Administración Pública & 22 \\
\hline Servicios a empresas & 22 \\
\hline Telecomunicaciones & 14 \\
\hline $\begin{array}{l}\text { Transportes y comunicacio- } \\
\text { nes }\end{array}$ & 12 \\
\hline Medios de Comunicación & 8 \\
\hline Educación e Investigación & 6 \\
\hline Energia y agua & 5 \\
\hline Industria de transformación & 5 \\
\hline Agroalimentación & 3 \\
\hline Comercio & 3 \\
\hline Editoriales & 3 \\
\hline Informática & 3 \\
\hline Librerías & 3 \\
\hline Artes gráficas & 2 \\
\hline Banca y finanzas & 2 \\
\hline Construcción & 2 \\
\hline Enseñanza & 2 \\
\hline Ocio y Cultura & 2 \\
\hline Sanidad & 2 \\
\hline Total & 154 \\
\hline
\end{tabular}

\subsubsection{Análisis de los requerimientos} de las empresas contratantes

Además de los estudios universitarios, las empresas requerían unas competencias y habilidades complementarias a los candidatos que quisiesen optar a los puestos ofertados.

\footnotetext{
${ }^{5}$ Los resultados fueron publicados íntegramente en Moreiro (2001).
} 


\begin{tabular}{|l|c|}
\hline Requerimientos explícitos & No. de ofertas \\
\hline Servicio Militar Cumplido & 2 \\
\hline Idiomas & \\
\hline Inglés & 46 \\
Francés & 5 \\
\hline Conocimientos Informáticos & \\
\hline Sistemas Operativos & 21 \\
Lenguajes de Programación & 5 \\
Bases de Datos & 68 \\
Hojas de Cálculo & 19 \\
Procesadores de Texto & 46 \\
Otros & 26 \\
\hline
\end{tabular}

De los datos expuestos en las tablas anteriores se deriva que el perfil más demandado es el siguiente:

La mayor actividad se ha dado en los campos de Consultoría, Servicios a Empresas y Administración Pública. EL tipo de puesto más ofertado ha sido el de documentalista, seguido del de ayudante bibliotecario. Para desarrollar funciones correspondientes en clasificación y catalogación, selección de información especializada, búsquedas de ínformación en Internet, depuración de registros y de resultados de búsquedas, organización y gestión de bibliotecas, y mantenimiento de bases de datos. EL $34 \%$ de las ofertas exigían un nível alto de inglés. Mientras que el conocimiento de la lengua inglesa se ha requerido en casi el $40 \%$ de las ofertas hechas. En la mayoría de los puestos ofertados se requirió dominar la gestión de bases de datos y de procesadores de texto, junto a un conocimiento holgado de las tecnologías de la información y la comunicación (In- ternet, Intranet, HTML, e-mail) y de los sistemas de gestión documental (ABSYS, MARC, KNOSYS). Resulta curioso que las empresas exijan a estudiantes y titulados recientes experiencia profesional previa superior a un año en más de un $7 \%$ de las ofertas.

Abordamos ahora la parte de aquel estudio relativa a las competencias que las empresas esperaban hubiesen adquirido los alumnos a lo largo de la formación universitaria en Ciencia de la Información, desde una doble visión, la de las propias empresas en los requisitos para los puestos de trabajo ofertados y en la valoración del nível con que los alumnos llegaban a esos puestos y, por otro lado, en la adaptación que propios alumnos manifestaron respecto a las funciones que debían desarrollar, 
3.5.2 Cualidades de los Estudiantes y Titulados

Consideramos finalmente las cualidades y carencias encontradas por los responsables de la contratación de los estudiantes, Respecto al nível de salida de la universidad, aparecen estas características como las mejor valoradas por las empresas respecto a las competencias con que estudiantes y titulados llegan al mercado laboral:

- Buen nível de los conocimientos técnicos en Biblioteconomía y Documentación $(80 \%)$, con capacidad por tanto para desarrollar las funciones propias del procesamiento de la información,

- Aprendizaje correcto del uso de las nuevas tecnologías, que les permite llegar a las empresas con grandes habilidades tecnológicas $(82 \%)$, se destaca el buen manejo de herramientas ofimáticas (93\%) y el conocimiento suficiente de técnicas y software de Biblioteconomía y Documentación (83\%).

- Otra característica muy apreciada ha sido el conocimiento de otras lenguas (85\%). Si bien se contrapone en las carencias con que no siempre es del nível deseado. Tampoco todos los titulados y estudiantes están capacitados para trabajar en otras lenguas.

- Es común que hayan completado su formación con cursos largos, de post- grado o extracurriculares (49\%), en especial la formación extra que muchos aportan en el área de empresa (28\%). Se constata así el comienzo de la capacidad de aprendizaje continuo que esta profesión necesita a lo largo de toda la vida.

- Mucho se han valorado también las actitudes de flexibilidad, entusiasmo por la profesión y capacidad de trabajo (48\%).

\subsubsection{Carencias Observadas}

En la otra parte de la balanza aparecen deficiencias formativas tanto generales como específicas:

- Se observa cierto desequilibrio entre la formación universitaria y las necesidades de las unidades de información (6\%). Aunque el porcentaje es bajo, sirve como llamada de atención para aproximar los curricula a las necesidades reales. Por más que sea difícil adaptar la rápida evolución de las técnicas y tecnologías de nuestro campo a los planes de estudio universitarios.

- Más preocupante es la constatación de un nível de formación general bajo, y la necesidad de una base cultural más amplia (22\%). Los planes de estudios, durante los últimos aõos, se han hecho con un cuño de especialización, por lo que el nível cultural básico no ha quedado garantizado. Lo mismo se viene achacando a la formación con que llegan los estudiantes a la Universidad. 
Parece como si la cultura humanística se haya relegado a ser tan sólo un recuerdo nostálgico del pasado. Cuando lo que las empresas buscan con este requerimiento es espíritu lógico y filosófico, desde el que poder entender y participar en las exigencias del mundo actual.

- Mayor capacidad de iniciativa, y de habilidades creativas (15\%).

- Conocimiento y uso altos de idiomas, en especial del inglés (18\%). Sin duda alguna, es una de las carencias cuya disminución persigue la formación universitaria española,

- Conocimiento insuficiente de entornos tecnológicos concretos (14\%). Lo cual resulta casi utópico, dada la continua aparición de software de aplicación documental. La Universidad no puede enseñar y practicar com todos los programas existentes en el mercado. En este caso deben valorarse más las habilidades informáticas adquiridas durante la carrera, que el conocimiento exhaustivo de cuantos programas existen en el mercado.

- Manejo de fuentes de información especificas: jurídicas, económicas, sanitarias, actuariales (38\%). Surge este silencio, sin duda, de la imposibilidad de atender en unos estudios de carácter general a todas las aplicaciones concretas de fuentes de información. La variedad de campos del saber hace imposible una especialización que aspiraria a ser casi universal.
- Finalmente aparece un grupo de observaciones referidas a deficiencias en las Técnicas de presentación y elaboración de proyectos $(7 \%)$, así como en las estrategias de búsqueda de empleo (técnicas de entrevista, presentación del curriculum vitae) (15\%). Es curiosa la constatación de este déficit formativo, cuando alguna disciplina incluye estos temas en su programa, y se imparten con la suficiente atención teórica y práctica. Algo similar sucede con el conocimiento de la gestión empresarial (9\%).

\section{COMPARACIÓN ENTRE LA RELACIÓN DE COMPETENCIAS DEFINIDAS PARA MERCOSUR Y PARA LA UNIÓN EURO- PEA}

Tanto la Unión Europea como MERCOSUR muestran un claro interés en aplicar políticas del conocimiento, que sin duda tienen una gran repercusión en los países que integran estas uniones. La ventaja europea proviene del mayor desarrollo temporal, legislativo y economico de su organización, más veterana, y por ello con una perspectiva más amplia de las aspiraciones y necesidades. Los planes para el desarrollo de la Sociedad de la Información en Europa cumplen estas finalidades: (CARIDAD, 1999)

1. Creación de infraestructuras transeuropeas que den acceso amigable a las informaciones referentes a los hechos eco- 
nômicos y sociales mundiales. Se enfatiza en la globalización.

2. Creación de nuevos mercados y complejos con el objetivo básico de que la nueva Sociedad de la Información obtendrá más recursos de la producción de bienes inmateriales que de los provenientes de bienes tradicionales.

3. La economía actual se basa en la creación de bienes y explotación de contenios informativos, hecho predominante en el Sigla XXI.

4. La Europa de la Unión se encuentra encaminada a la creación de un espacio común del conocimiento.

En este marco que destaca el papel social y económico de la información cobra mayor importancia los rasgos que definen el campo de actuación de los profesionales que atienden y entienden de información.

\subsection{Categorización de Competencias} Deseables y Exigibles a un Profesional (CCDEP) egresado de una Universidad en el Área de Bibliotecología/Ciencia de la Información en el MERCOSUR

El perfil de las competencias del profesional de la información fue abordado, en el caso de MERCOSUR, durante el IV Encuentro de Directores de Escuelas de Bibliotecología y Ciencia de la Información del MERCOSUR que se celebró en Monte- video, del 24 al 27 de mayo de $2000 .{ }^{6}$ En el debate participaron ponentes de todos los países que forman esa Unión mercantil supranacional, es decir:

- Argentina;

- Brasil;

- Paraguay;

- Uruguay;

- además de contarse con la presencia de Chile, país extracomunitario.

EL documento derivado fue sancionado por todos ellos, y categorizó las competencias requeridas por los profesionales de la información en el momento en que se abren al mercado laboral, una vez terminados los estudios universitarios. La sistematización de la lista resultante, de cuarenta y tres competencias, se mostró en cuatro grandes subdivisiones:

(A) Competencias en comunicación y expresión (6);

(B) Competencias técnico-cientí-ficas (16);

(C) Competencias gerenciales (9);

(D) Competencias sociales y políticas (12).

En esta categorización de competencias no se establecen diferencias entre los

\footnotetext{
${ }^{6}$ Anexo 3. IV Encuentro de Directores de Escuelas de Bibliotecología y Ciencia de la Información del MERCOSUR. Montevideo, 24 al 27 de mayo de 2000 (VALENTIM, 2001, p. 17-21)
} 
campos de competencia y aquellos propios de las aptitudes, si bien se incluyen algunas de estas en la enumeración de las competencias sociales y políticas.

A. Competencias en comunicación y expresión:

A.1. Formular y gestionar proyectos de información.

A.2. Aplicar técnicas de marketing, liderazgo y de relaciones públicas.

A.3. Capacitar y orientar a los usuarios para el mejor uso de las unidades de información y sus recursos.

A.4. Elaborar productos de información (bibliografias, catálogos, guías, índices, DSI, etc.).

A.5. Ejecutar procedimientos automatizados propios de un entorno informatizado.

A.6. Planificar y ejecutar estudios de usuarios / clientes de la información y formación de usuarios / clientes de la información.

B. Competencias técnico-científicas:

B.1. Desarrollar y ejecutar el procesamiento de documentos en distintos soportes en unidades, sistemas y servicios de información.

B.2. Recolectar, registrar, almacenar, recuperar, y difundir la información grabada en cualquier medio para los usuarios de unidades, servicios y sistemas de información.

B.3. Elaborar productos de información (bibliografias, catálogos, guías, índices, DSI etc.).

B.4. Utilizar y diseminar fuentes, productos y recursos de información en diferentes soportes.

B.5. Reunir y valorar documentos y proceder a archivarlos.

B.6. Preservar y conservar los materiales albergados en las unidades de información.

B.7. Seleccionar y evaluar todo tipo de material para las unidades de información.

B.8. Buscar, registrar, evaluar y difundir la información com fines académicos y profesionales.

B.9. Ejecutar procedimientos automatizados propios de un entorno informatizado.

B.10.Planificar y ejecutar estudios de usuários / clientes de la información y formación de usuários / clientes de la información.

B.11 Planificar, constituir y manejar redes globales de información.

B.12. Formular políticas de invéstigación en Bibliotecología y Ciencia de la Información. 
B.13. Realizar investigaciones y estudios sobre desarrollo y apli-cación de metodología de elaboración y utilización del conocimiento registrado.

B.14. Asesorar e intervenir en la elaboración de normas jurídicas en Bibliotecología y Ciencias de la Información.

B.15. Asesorar en la tasación de colecciones bibliográficos documentales.

B.16. Realizar peritajes referidos a la autenticidad, antigüedad, procedencia y estado de materiales impresos de valor bibliofilico.

\section{Competencias gerenciales:}

C.1. Dirigir, administrar, organizar y coordinar unidades, sistemas y servicios de informaclon.

C.2. Formular y gestionar proyectos de información.

C.3. Aplicar técnicas de marketing, liderazgo y de relaciones publicas.

C.4. Buscar, registrar, evaluar y difundir la información con fines academicos y profesionales.

C.5. Elaborar productos de información (bibliografias, catálogos, guías, índices, DSI, etc.).

C.6. Asesorar en el planeamiento de los recursos económicos, financieros y humanos del sector.
C.7. Planificar, coordinar y evaluar la preservación y conservación del acervo documental.

C.8. Planificar y ejecutar estudios y formación de usuarios/clientes de la información.

C.9. Planificar, constituir y manejar redes regionales y globales de información.

D. Competencias sociales y políticas:

D.1. Seleccionar y evaluar todo tipo de material para las unidades de información.

D.2. Buscar, registrar, evaluar y difundir la información con fines açadémicos y profesionales.

D.3. Asesorar e intervenir en la formulación de políticas de información.

D.4. Asesorar en el planeamiento de los recursos económico financíeros y humanos del sector.

D.5. Planificar y ejecutar estudios de usuarios/clientes de la información y formación de usuaríos/clientes de la información.

D.6. Promover una actitud crítica y creativa respecto a la reso-lución de problemas y cuestiones de información.

D. 7. Fomentar una actitud abierta e interactiva con los diversos actores sociales (políticos, empresarios, educado- 
res, trabajadores y profesionales de otras áreas, instituciones y ciudadanos en general).

D.8. Identificar las nuevas demandas sociales de información.

D.9. Contribuir a definir, consolidar y desarrollar el mercado laboral en el área.

D. 10. Actuar colectivamente con sus pares en el ámbito de las instituciones sociales, con el objetivo de la promoción y la defensa de la profesión.

D.11. Formular políticas de investigación en Bibliotecología y Ciencia de la Información.

D.12. Asesorar e intervenir en la elaboración de normas jurídicas en Bibliotecología y Ciencias de la Información.

\subsection{EL Proyecto de "Desarrollo de las} Eurocompetencias en Información y Documentación"

Este proyecto, conocido por las siglas DECIDOC, se disenó con el fin de "conseguir un conocimiento mejor de las competencias en el campo profesional de la información y documentación, y una transparencia de las competencias clave adaptadas al desarrollo tecnológico, a la competitividad de las empresas y a las necesidades del mercado de trabajo" (COMISSION EUROPĖENNE, 2001). Las actividades para plasmarlo empezaron en 1995 dentro del programa europeo Leonardo Da Vinci a iniciativa del Consejo Europeo de Asociaciones de Información y Documentación (ECIA), integrado por asociaciones profesionales de nueve países de la Unión Europea:

- Alemania (DGI)

- Bélgica (ABD-BVD)

- Espana (SEDIC)

- Finlandia (Tietopalveluseura)

- Francia (ADBS, que en este proyecto actuó como líder)

- Italia (AIDA)

- Portugal (INCITE)

- Reino Unido (ASLIB)

- Suecia (TLS)

A las que además se incorporaron estas otras asociaciones profesionales:

- ASA de Suiza.

- Inform. Doc. Rom. de Rumania.

- SKIP de la República Checa.

La elaboración se hizo desde el Référentiel des métiers-types et compétences des professionnels de l'information et documentation de la ADBS y desde la Relación de conocimientos y técnicas aplicables a las funciones de los profesionales de la información y la documentación de la SEDIC (1996), aunque este último se realizó partiendo del documento 
de la asociación francesa. El proyecto identificó treinta campos en los que se pueden ejercer las competencias. Estos campos aparecen divididos en cuatro grupos:

1. Los diez primeros campos tienen que ver con los conocimientos teóricos y prácticos fundamentales. Se trata de las competencias permanentes de la profesión: identificación y selección de las fuentes de información, tratamiento documental, búsquedas documentales, los servicios de difusión.

2. Los campos $11 / 19$ agrupan las competencias relativas a la comunicación.

3. Mientras que el grupo de campos 20/29 se refiere a la gestión de la organización. El mismo referencial advierte que estas competencias no son exclusivas de esta profesión, pero señala que tiene unas aplicaciones específicas en nuestro campo.

\section{Los conocimientos externos a nu-} estro campo constituyen el cuarto y último grupo de competencias, determinado de forma genérica en el campo 30. El listado de este grupo presenta la relación más extensa, debido a que se ocupa de conocimientos que pueden ser útiles en contextos concretos.

Además de las competencias, la relación europea incluye las quince aptitudes básicas para la profesión. Muchas de ellas no son exclusivas de nuestro campo, pero su "importancia puede ser decisiva en el perfil de un buen profesional de la IDOC" (ECIA, 2000, p. 14). ${ }^{7}$

EL mismo individuo no puede llegar a los mismos niveles de competencia en cada uno de los campos, debido a que la competencia general de una persona es el resultado de combinar varias competencias a diversos niveles. En su adquisición, las competencias ofrecen una graduación en cuatro niveles sucesivos, correspondientes con los niveles que marcan los especialistas en formación para los objetivos pedagógicos:

1. En el nível básico o de sensibilización, se conoce la existencia y la naturaleza de los elementos de una determinada función, y se manejan los términos con los que identificar los problemas.

2. En el nivel de conocimiento práctico, ya profesional, se aprende a manejar aplicaciones básicas, alcanzándose la capacidad de efectuar trabajos técnicos.

3. En el tercer nível, de domínio de aplicaciones, se controlan las distintas técnicas, y se alcanza la capacidad de desarrollar nuevas aplicaciones en el oficio.

4. En el nivel de domínio métodológico, se llega a planificar estratégicamente y a concebir nuevas aplicaciones y productos.

\footnotetext{
7 Se ha traducido ya a siete lenguas europeas: inglés, francés, alemán, checo, portugués, rumano y espanol. En el caso del espanol: Relación de eurocompetencias en información y documentación. Madrid: SEDIC, 2000.
} 
Se exponen a continuación los treinta encabezamientos de los campos de competencia y las quince aptitudes del referencial:

1. Interacción con los usuarios y los clientes.

Analizar e interpretar las prácticas, las demandas, las necesidades y las expectativas de los usuarios y clientes, actuales y potenciales, y desarrollar su cultura de la información ayudándoles a hacer el mejor uso de los recursos disponibles.

2. Conocimiento del entorno profesional de la Información y Documentación.

Orientarse en el entorno profesional nacional e internacional de la información y documentación, así como en su media político, económico e institucional.

3. Conocimiento del marco jurídico y administrativo europeo de la gestión de la información.

Aplicar las disposiciones y los procedimientos legales y reglamentarios tanto de ámbito nacional como los emanados de la Unión Europea relativos a la actividad de información y documentación.

4. Identificación y validación de fuentes de información.

Identificar, evaluar y validar informaciones, documentos y sus fuentes, tanto internos como externos.

5. Gestión de colecciones y rondas
Elaborar y aplicar criterios de selección, de adquisición y de eliminación de documentos que permitan constituir y organizar colecciones de documentos de toda naturaleza o rondas de archivos, conservarlos haciéndolos accesibles, desarrollarlos teniéndolos al día y expurgarias de elementos que se han convertido en inútiles, siguiendo la evolución de las necesidades de los usuarios.

6. Tratamiento fisico de documentos.

Definir y aplicar métodos y técnicas para ordenar, proteger, conservar, restaurar soportes documentales de cualquier naturaleza.

7. Análisis y representación de la información.

Identificar y representar en el lenguaje documental adaptado o en otro sistema simbólico el contenido semántico de un documento o de una colección de documentos.

8. Organización y almacenamiento de la información.

Organizar y estructurar los datos relativos a la descripción de documentos y colecciones de documentos en cualquier soporte; crear y explotar las herramientas de acceso a los datos, documentos o referencias.

9. Búsqueda de información.

Buscar y recuperar la información por métodos que permitan dar respuesta a las 
expectativas de los demandantes en condiciones optimas de coste y tiempo.

10. Elaboración y difusión de la información.

Hacer disponibles y explotables las informaciones tratadas y facilitar el uso mediante el suministro de productos y servicios documentales.

11. Comunicación oral.

Comprender los mensajes recibidos oralmente en el idioma de trabajo; expresarse oralmente en diferentes entornos profesionales y adaptar el mensaje a su interlocutor o interlocutores.

\section{Comunicación escrita}

Leer y comprender documentos escritos en el idioma de trabajo; expresarse y hacerse comprender por medios escritos en diferentes entornos profesionales.

13. Práctica de una lengua extranjera

Comprender y utilizar la lengua considerada, tanto en la vida cotidiana como en las actividades profesionales.

14. Comunicación visual y sonora

Transmitir informaciones haciéndose entender mediante imágenes y sonidos (gráficos, curvas, diagramas; mapas, planos, fotografias, películas, vídeos, batidas sonoras).

15. Comunicación interpersonal
Hacer más fáciles y más eficaces las relaciones con los indivíduos y los grupos en toda clase de situaciones y contextos: intercambio, negociación, formación.

16. Comunicación institucional

Concebir y realizar operaciones de comunicación tanto internas (dentro del organismo) como externas.

17. Tecnologías de la información: informática

Utilizar y poner en práctica métodos, técnicas y herramientas informáticas (hardware o software) para la implantación, desarrollo y explotación de sistemas de información.

18. Tecnologías de la información: telecomunicaciones

Utilizar y poner en práctica tos métodos, las técnicas y las herramientas (hardware o software) para la implantación, desarrollo y explotación de sistemas de telecomunicación.

19. Técnicas de producción y edición

Producir o reproducir documentos en cualquier soporte (papel, microficha, magnético, óptico) con vistas a su difusión.

20. Técnicas de gestión administrativa

Garantizar el mantenimiento de la administración general, la gestión administrativa y el apoyo logístico de la actividad de un organismo. 
21. Técnicas de marketing

Analizar y situar la actividad en un contexto estratégico y de competencia; promover dicha actividad elaborando y poniendo a punto las herramientas de trabajo apropiadas para la captación del mercado.

22. Técnicas comerciales.

Establecer y mantener relaciones con clientes o socios con el fin de vender productos y servicios.

\section{Técnicas de adquisición}

Adquisición de los productos documentos o prestaciones, en función de normas vigentes para su gestión y de una política de adquisiciones establecida.

24. Técnicas de gestión microeconómica

Controlar y optimizar de forma permanente los recursos del organismo y su utilización.

25. Técnicas de instalación, acondicionamiento y equipamiento

Organizar fisicamente el espacio de trabajo, de conservación, de la recepción del público, con vistas a proporcionar los servicios que se esperan.

26. Técnicas de planificación y gestión de proyectos

Prever, organizar, gestionar y llevar a buen término un proyecto técnico integrando las limitaciones del entorno: huma- nas, económicas, de calendario, reglamentarias.

27. Técnicas de diagnóstico y evaluación

Identificar los puntos fuertes y débiles de una organización, de un producto o de un servicio, establecer y utilizar indicadores, elaborar soluciones para mejorar la calidad.

28. Técnicas de gestión de recursos humanos

Asegurar la integración, la eficacia y el bienestar del personal de una unidad de trabajo, aplicando la legislación y la reglamentación en vigor, respetando los objetivos de la empresa, favoreciendo el desarrollo personal y profesional de los individuoso.

\section{Técnicas de formación}

Concebir y ejecutar una acción o un plan de formación inicial o continua

30. Todos los conocimientos útiles para la gestión de la información

Sacar provecho de los conocimientos ofrecidos por disciplinas ajenas a la información para resolver mejor los problemas encontrados en el ejercicio de actividades de información y documentación y hacer frente a situaciones profesionales determinada. Por ejemplo, una determinada cultura jurídica es útil para discutir o negociar un contrato con los propietarios de derechos sobre fuentes de información, una cultura 
histórica para evaluar un archivo, una cultura estadística para poner a punto indicadores de flujo de la comunicación científica. Las aptitudes derivadas de estos conocimientos son evidentemente diferentes según las disciplinas a las que pertenecen, $y$ sus niveles se deben apreciar teniendo en cuenta la especificidad de cada uno.

\subsubsection{Relación de Aptitudes}

Com referencia a las aptitudes, la Relación ofrece una lista comentada de solo quince que son comunes a muchas profesiones intelectuales, y por lo tanto no son monopolio de la IDOC. Precisamente por esta razón su descripción aparece bastante breve. Sin embargo, son de importancia extrema:

\section{Capacidad de adaptación}

2. Capacidad de análisis

3. Sentido de anticipación

4. Capacidad de comunicación

5. Espíritu crítico

6. Curiosidad intelectual

7. Capacidad de decisión

8. Capacidad de escuchar

9. Capacidad para el trabajo en equipo

10. Capacidad de iniciativa

11. Sentido de organización

12. Sentido pedagógico
13. Perseverancia

14. Rigor

15. Capacidad de síntesis

EL objetivo final de este proyecto no se queda en identificar las competencias, ya que con ellas persigue establecer el marco desde el cual certificar a los profesionales desde esas competencias a nivel europeo, además de programar en este contexto la formación a distancia necesaria para perfeccionarlas. Jean Meyriat (SEDIC, 2000) ha fijado en tres puntos la continuidad del proyecto de las Eurocompetencias, que él considera la base para las aspiraciones a una certificación de verdadero alcance europeo, común para todos los países, aunque con aplicaciones diversas dependiendo del contexto propio de cada país:

1. La continua puesta al dia de esta relación, ya que en nuestra disciplina las técnicas evolucionan muy rápidamente.

2. La ampliación del uso y difusión de esta relación, no sólo en Europa, sino que además puede ser útil en otros continentes.

3. La elaboración de una guia para la evaluación de competencias, objetivo dificil de alcanzar, pero necesario para aplicarse en los procesos de certificación que se están llevando a cabo en diversos países europeos. 


\subsection{Comentarios a ambas propuestas}

Comparativamente y con carácter general se aprecia una mayor descripción de los conocimientos implicados en cada competencia en el Euroreferencial lo que contribuye a desambiguar la significación de cada competencia. Cada Eurocompetencia es denominada terminológicamente, para ser luego descrita a través de verbos representativos de las acciones que implica. Mientras que la Categorización de Competencias de MERCOSUR prefiere tan solo enumerarlas mediante acciones verbales. A la hora de distinguir entre competencias y aptitudes, aparecen sistematizadas de modo bien diferenciado en el caso europeo, mientras que, en el caso sudamericano, aparecen embutidas en el listado de las competencias, cuando no omitidas.

La Categorización de Competencias de MERCOSUR se presenta agrupada explícitamente en cuatro clasificaciones, apartando de esta manera gran claridad al esquema expositivo. En el caso europeo el listado de las competencias se presenta de modo continuo, si bien se introduce un comentario previo que indica que se establecen cuatro grupos en los treinta campos, pero no deja de ser una clasificación implícita, menos evidente a la hora de consultar el listado. Aún así, la superestructura en que se sistematizan las competencias presenta un gran paralelismo en ambos casos. Puede observarse que entre la primera subdivisión de MERCOSUR, "Competencias en Comunicación y Expresión”, y la europea, "Competencias Relativas a la Comunicación", hay coincidencia hasta en el nombre. Lo mismo sucede con las "Competencias Gerenciales", que en caso europeo se denominan de "Gestión de la Organización". Mayor diferencia denominativa y conceptual se percibe entre el grupo de "Competencias Técnico-Científicas" americanas y los "Conocimientos Teóricos y Prácticos Fundamentales" que prefiere el listado de la Unión Europea. La mayor originalidad de la relación de MERCOSUR se da en el caso de las "Competencias Sociales y Políticas", cuyas especificaciones se incluyen mayoritariamente, en el caso de la relación europea, dentro de las "Competencias Relativas a la Gestión y a los Conocimientos Teóricos y Prácticos Fundamentales", sin olvidar la posible presencia de estos contenidos también en las conocimientos externos a nuestro campo.

Si profundizamos un poco en el análisis concreto de las competencias podemos observar estas diferencias y similitudes:

En el caso europeo los campos que tienen que ver con los conocimientos teóricos y prácticos fundamentales están encabezadas por la "interacción con los usuarios y los clientes", para apuntalar la importancia del servicio a los usuarios en nuestra profesión. Sin duda en la propuesta americana subyace este interés, pero no 
se explícita, empero la importancia del papel social de las competencias profesionales esté muy destacado sobre el caso europeo. La descripción del servicio a los usuarios en las directrices de MERCOSUR queda parcialmente incluida en las entradas 5,7 y 8 del campo de competencias sociales y políticas, así como en la 3 y 6 de comunicación y expresión. De otra parte, la inclusión de un Conocimiento del entorno profesional, en el caso europeo, no encuentra paralelismo en las definidas en CCDEP, si bien esta propuesta se recoge tangencialmente al hablar de la actuación colectiva para la defensa y promoción de la profesión dentro de las competencias sociales y políticas.

En el caso de las Competencias técnico-científicas de MERCOSUR, la primera de las descritas (B 1) viene a ser la síntesis de lo que luego se desarrolla en ese gran apartado, en cuya enumeración se hace dificil seguir el orden del proceso técnico. La propuesta europea sigue en la parte técnica el orden clásico del procesamiento de la información en la cadena documental. Sin duda al optarse por este sistema procesual se ayuda a la comprensión de lo que requiere cada competencia de este apartado. Sin duda haber optado por una redacción indicativa de las competencias lleva a posibles entrecruzamientos comprensivos como es el caso de las competencias de MERCOSUR B5 y B7: ¿qué diferencia hay entre valorar documentos $y$ evaluar todo tipo de material? Una mayor explicación contribuiría a concretar el significado. Un caso parecido de sinonimia lo encontramos entre la B2 y la B4, e incluso con la B8, cuando se añaden con fines académicos o profesionales, pero que también podrían ser fines de ocio o de interés económico o cultural. Por ello es mejor adoptar una postura neutra, no restrictiva del uso que vaya a darse a la información tratada, ya que este depende de los usuarios. Y si la aplicación profesional y científica ennoblece nuestro trabajo, bien es cierto que el uso depende de la voluntad y ética de los usuarios y que Internet ha venido también a desdibujar los límites del uso de la información. Se repite la dificultad para asegurar un significado diferente entre la propuesta que hace la entrada B 15 y la de la B16.

Nos llama también la atención que se incluya en este apartado una descripción idéntica, la B9, a la que aparece en la A5 de las "Competencias en Comunicación y Expresión": "Ejecutar procedimientos automatizados propios de un entorno informatizado", más determinadas en el caso europeo. Creemos que se trata de una competencia comunicativa más que técnica. Lo técnico se viabiliza mediante la expresión y la comunicación digitales, pero no solo lo técnico de nuestro sector de trabajo. Sin duda alguna el interés de la Unión europea por acentuar este caso y el de la expresión, especialmente en lenguas extranjeras, ma- 
nifestados dentro de unas amplias (nueve campos) competencias relativas a la comunicación manifiesta la preocupación porque la información fluya eficazmente dentro de una comunidad multilingüe y pluricultural, y también entre la Unión y el resto de países. A la luz de los resultados obtenidos por mediciones propias es un acierto incluir los apartados relativos a la Comunicación, pues creemos que deben considerarse siempre, ya que nuestra intermediación es comunicativa y cualquier procedimiento técnico-profesional irá al fracaso sin contar con un buen nível expresivo. La importancia de que el profesional de la información sea capaz de comunicarla, haciéndose enteder y comprendiendo previamente los mensajes de los usuarios resalta el valor que en este sector tiene un adecuado nível cultural, el dominio de los temas de información general y la calidad de la expresión tanto oral como escrita. Mientras que en el resto de los campos de las Competencias técnico-científicas hay un gran paralelismo entre las dos propuestas.

Lo más peculiar, por su carácter reivindicativo y de aplicación social de nuestra profesión, viene en el caso de MERCOSUR de la mano de las "Competencias Sociales y Políticas", reflejo de la función que los sistemas de información deben cumplir en el fomento de la educación, la cultura y el desarrollo. Sin duda, destacar este interés con un apartado específico que se especifica en doce entradas, mues- tra el papel al que la información está llamada en estos países, y la confianza de los profesores en que las futuras generaciones de profesionales sean capaces de contribuir a modificar la realidad que les rodea, siempre con el reto de aspirar a una auténtica implantación del MERCOSUR.

En ambas relaciones constan como cimientos del edificio profesional de la información una serie de capacidades relacionadas con:

- El conocimiento técnico.

- La gestión y dirección.

- Las habilidades de comunicación y expresión lingüística.

- Las habilidades informáticas.

- Considerándose, además, una serie de actitudes personales y habilidades creativas.

\section{REFERENCIAS}

AMERICAN ASSOCIATION OF SCHOOL LIBRARIAN. Association for Educational Communicatlons and Technology. Information Power. Guidelines for School Library Media Programs. Chicago: ALA; Washington: Association for Educational Communications and Technology, 1988.

AMERICAN LIBRARY ASSOCIATION. Human resources in the 1990's. Library Personnel News, v. 41, n. 2, p. 17-19, 1990.

AMERICAN 2000: An education strategy. Sourcebook. Washington: US Dept. of Education, 1991. 
AROT, D. Les valeurs professionnelles du bibliothécaire. Bulletin des bibliothèques de France, v. 45, n. 1, p. 33-41, 2000.

BARDEN, P. Training and development for library and information workers for the future: a manifesto. Librarian Career Development, v.5, n.1, p. 30, 1997.

CARIDAD, M. Planes de la Unión Europea para alcanzar en política del conocimiento. In: (coord.) La Sociedad de la Información: política, tecnología e industria de los contenidos. Madrid: Editorial Centro de Estudios Ramón Areces, 1999.

COMMISSION EUROPÈENNE. Project DECIDoc: rapport final. Bruxelles: Commission Européenne. Direction Général XXII, 2001.

COOPER, M.; LUNIN, L. F. Education and training of the information professional. Annual Review of Information Science and Technology, v. 24, p. 295-341, 1989.

COUNCIL OF EUROPE. New professional profiles and competencies for information professionals and knowledge workers operating in cultural industries and institutions: results of lhe consultation process. December 1998 - February 1999. Strasbourg: Council of Europe, 1999.

CRONIN, B. et al. The emergent Market for Information Professionals: Educational Opportunities and Implications. Library

Trends, v. 42, n. 2, p. 257-276, 1993.

The transition years: new initiatives in lhe education of professional information workers. London: ASLIB, 1983.

DAVENPORT, T.; PRUSAK, L. Working Knowledge: how organizations manage what they know. Boston: Harvard Business School Press, 1998.

DESCHAMPS, J. Nouvelles competences en information documentaire et technologie de l'information: etude des offres d'emploi du Bulletin des postes vacants de l'ESID sur quatre années de référence. Geneve:
Ecole Supérieure d'Information Documentaire, 1999.

ECIA. Euroréférentiel de l'information et documentation/ Euro guide Library in information services/ Relación de Eurocompetencias en información y documentación. Bruselas: ECIA, 2000.

EURO-CHAMBRES. FutureProft: Flexible training profiles/curricula meeting the demands of lhe future, taking account of key qualifications required in Ihe ITC fields of multimedia / EDP, telecommunication, marketing / advertising. 1999. Disponível em:

<http://www.webb.co.at/futureprof/summary .html> Acesso em: 20 ago. 2003.

HORTON, F.W. (ed.). Understanding US information policy: the infostructure handbook. Washington: Information Industry Association, 1982.

INSTITUTE OF INFORMATION SCIENTISTS. Criteria for information science. Disponível em: <http://www.iis.org.uk/membership/Criteria. html>. Acesso em: 20 ago. 2003.

KUTSCHER, R. The major trends. Occupational Outlook Quarterly, v. 34, p. 3-7, 1990.

LEANNONT, C.; VAN HOUTEN, S. Placements and salaries 1989: Steady on. $\mathrm{Li}$ brary Journal, v. 115, n. 17, p. 46-52, 1990.

LINE, M. B. Le métier de bibliothécaire: un ensemble de pratiques confuses et Discontinues. Bulletin des bibliothèques de France, v. 43, n. 2, p. 46, 1998.

MORAN, B. Virtual realists: librarians in a time of transition. In: LAUNC-CH 1999 SPRING CONFERENCE. GET REAL: VIRTUAL REALITY AND EVERYDAY LIFE. Chapel Hill: 1999. Disponível em: <http://www.unc.edu/lib/launcch/moranArt.h tml> Acesso em: 20 ago. 2003. 
MOREIRO, J. A. Figures on employability of Spanish Library and Information Science graduates. Libri, v. 51, n.1, p. 27-37, 2001.

Mercado de trabajo y competencias profesionales en Biblioteconomía y Documentación: técnicas aplicables a su investigación. Informação\&Sociedade, v.8, n.1, p. 124-151, 1998.

SCHLESSINGER, B.; SCHLESSINGER, J.; KARP, R. Information Science / Library Science Education Programs in the 1990s: a not-so-modest proposal. Library Administration and Management, v. 5, p. 16-19, 1991.

SEDIC. Relación de conocimientos y técnicas aplicables a las funciones de los profesionales de la información y la documentación. Madrid: SEDIC, 1996.

SEDIC. Relación de Eurocompetencias en Información y Documentación. Madrid: SEDIC, 2000.

\section{SPECIAL LIBRARIES ASSOCIATION.} Special Committee on Competencies for Special Librarians. Competencies for special librarians of the $21^{\text {st }}$ century. Special Library Association, 1996. Disponível em: $<$ http//www.sla.org/professional/competenc y.html> Acesso em: 20 ago. 2003.

SPIEGELMAN, B. M. (ed.). Competencies for special librarians for 21st Century. Washington: Special Libraries Association, 1997.

TEJADA ARTIGAS, C. Adecuación de los planes de estudio de la Diplomatura en Biblioteconomía y Documentación a las demandas del mercado de trabajo: el caso concreto del nuevo plan de estudios de la Escuela Universitaria de Biblioteconomia y Documentación de la Universidad Complutense de Madrid. Madrid: 2002. Tese (Doutorado) - Programa de Doctorado en Documentación, Departamento de Biblioteconomía y Documentación, Universidad Carlos III, Madrid, 2002.
TENNANT, R. Digital Libraries: skills for the New Millenium. Library Journal, v. 124, n. 1, p. 38, 1999.

TFPL. Skills for knowledge management: a briefing paper. 1999. Disponível em: <http://www.tfpl.com/areas_of_expertise/ knowledge_management/KM_skills_resear ch/KM_skills_executive_summary/KM_skill s. Ndf> Acesso em: 20 ago. 2003.

VALENTIM, M. Introdução. Profissional da Informação: formação, perfil e atuação profissional. In: _. (org.) Profissionais da Informação: formação, perfil e atuação profissional. São Paulo: Polis: 2000:

WARD, S. Information professionals for the next millennium. Journal of Information Science, v. 25, n. 4, p. 239-247, 1999. 


\section{Jose Antonio Moreiro \\ Universidad Carlos III de Madrid \\ jamore@bib.uc3m.es}

\section{Carlos Miguel Tejada Artigas \\ Universidad Complutense de Madrid \\ tejada@caelo.eubd.ucm.es}

\section{Title}

Required competences for the Information Professions performance: value attributes to Mercosul's and European Union's related lists

\begin{abstract}
The Information Society requires new attitudes and competences which are necessary for a well-developed professional, having as reference the professions that deals with information as well as the ones related to the entrepreneurs need, concerning the treatment of the needed and used information. The today's required competences, especially in Information Science, constitute an important subject for the university development discussion, mainly at the graduation courses. The article presents the competences of professionals from Librarianship and Documentation, following the recommendations of the European Council, the observed lacks of the area, the competences defined by the Mercosul and the European Union, as well as a project for the development of those competences; known as the abbreviation DECIDOC.
\end{abstract}

\section{Keywords}

Competences; Information Professional; Mercosul; European Union; DECIDOC.

\section{Título}

Competências requeridas para o exercício das Profissões da Informação: valoração das listas relacionais do Mercosul e da União Européia

\section{Resumen}

La Sociedad de la Información exige nuevas actitudes y desarrollo de competencias necesarias para un buen desarrollo profesional; teniendo como referencia las profesiones que trabajan con información, así como, en relación a las necesidades empresariales referentes al tratamiento de la información que las necesitan y las utilizan. Hoy, las competencias requeridas, específicamente en el área de la Ciencia de la Información, son una necesidad a ser pensada por parte de la formación universitária, principalmente en los cursos de graduación. Son presentados en el texto, las competencias profesionales en Bibliotecología y Documentación, de acuerdo con recomendaciones del Consejo de la Europa; las carencias observadas en el área; las competencias definidas para el Mercosur y la Unión Europea; así como un proyecto para el desarrollo de esas competencias, conocido por la sigla DECIDOC.

\section{Palabras Clave}

Competencias; Profesional de la Información; Mercosur; Unión Europea; DECIDOC 